\title{
Erratum to: Flood Forecasting and Decision Making in the new Millennium. Where are We?
}

\author{
Ezio Todini ${ }^{1}$
}

Published online: 10 July 2017

(C) Springer Science+Business Media B.V. 2017

\section{Erratum to: Water Resour Manage \\ DOI 10.1007/s11269-017-1693-7}

Due to a misplaced index, the Fig. 3 of article "Flood Forecasting and Decision Making in the new Millennium. Where are We?" by E. Todini, was incorrectly drawn and misinterpreted in the text. Although this misinterpretation doesn't alter the overall assert that the ensemble approach does not allow for a correct estimation of the predictive density, it was felt necessary to provide the relevant correction.

The text from end of page 6 and in page 7:

"As can be seen from Fig. 3, in both calibration (left) and validation (right) periods most of the prediction errors fall in the two lowest quantiles $(75 \%$ in calibration and $\sim 85 \%$ in verification), indicating that, similarly to what could be visualized in Fig. 2 a large quantity of predictions underestimate the flood values. Moreover, it should be noticed that the large majority of frequency values shown as a histogram for the 18 ranges lie far from the expected value (solid horizontal line) and outside the confidence limits estimated with the Wilson (1927) bounds (dashed horizontal lines).

The online version of the original article can be found at http://dx.doi.org/10.1007/s11269-017-1693-7

Ezio Todini

ezio.todini@gmail.com

1 BiGeA, University of Bologna, Via Zamboni, 67, 40126 Bologna, Italy 
The original Fig. 3 and its caption.
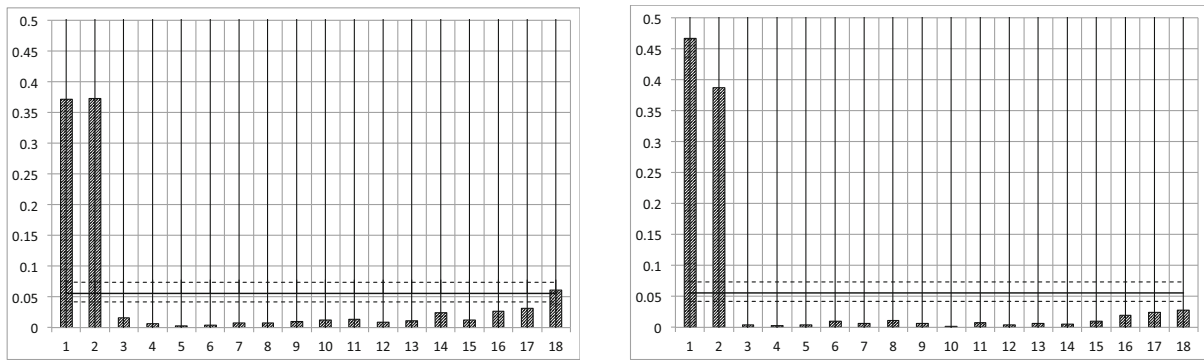

Fig. 3 Observed frequencies of prediction errors falling into the 18 equiprobable ranges $\left(0 \div \frac{1}{m+1}\right)$, $\left(\frac{1}{m+1} \div \frac{1}{m+1}\right), \ldots,\left(\frac{1}{m+1} \div 1\right)$ for operational flood forecasts at Pontelagoscuro based on 17 COSMO-LEPS ensemble members taken as a representation of predictive uncertainty quantiles. Instead of a uniform frequency distribution, both in calibration (left) and validation (right) the observed frequencies of errors tend to be highly concentrated on the low quantiles (underestimation) and most of the estimated frequencies lie out of the Wilson (1927) confidence intervals. “

Must correctly read:

"As already found by several authors (Hamill and Colucci 1997; Eckel and Walters 1998), in both calibration (left) and validation (right) periods the ensemble is under-dispersive and most of the prediction errors fall in the lowest and highest quantiles ( $75 \%$ in calibration and $\sim 85 \%$ in validation), indicating that a number of predictions, much larger than what expected from the ensemble spread, will highly underestimate or overestimate the flood values. Moreover, it should be noticed that the large majority of frequency values shown as a histogram for the 18 ranges lie far from the expected value (solid horizontal line) and outside the confidence limits estimated with the Wilson (1927) bounds (dashed horizontal lines).

Must be substituted by the following Fig. 3 and its caption
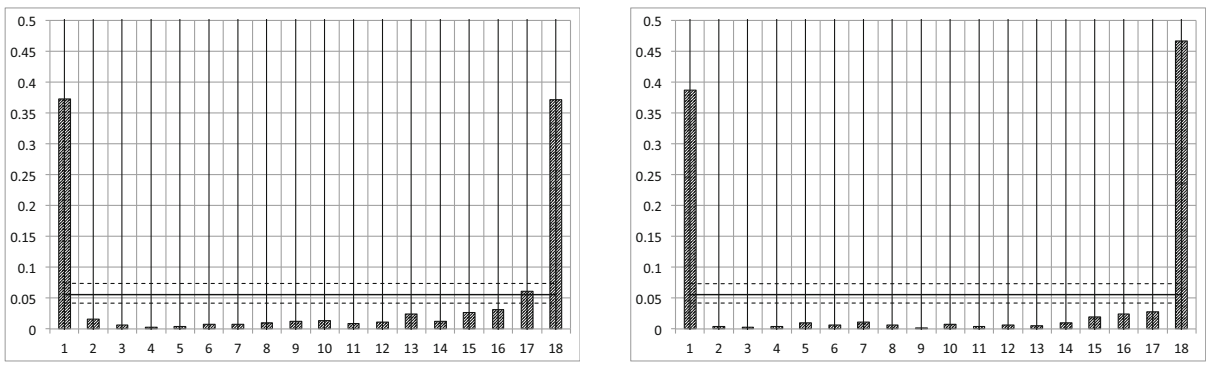

Fig. 3 Observed frequencies of prediction errors falling into the 18 equiprobable ranges $\left(0 \div \frac{1}{m+1}\right)$, $\left(\frac{1}{m+1} \div \frac{1}{m+1}\right), \ldots,\left(\frac{1}{m+1} \div 1\right)$ for operational flood forecasts at Pontelagoscuro based on 17 COSMO-LEPS ensemble members taken as a representation of predictive uncertainty quantiles. Instead of a uniform frequency distribution, both in calibration (left) and validation (right) the observed frequencies of errors tend to be highly concentrated on the lowest and highest quantiles and most of the estimated frequencies lie out of the Wilson (1927) confidence intervals. “ 